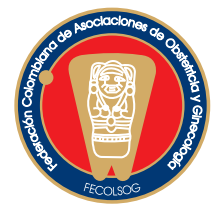

Reporte de caso

\title{
SÍNDROME DE AGLOSIA-ADACTILIA Y EXPOSICIÓN PRENATAL A MISOPROSTOL ¿RELACIÓN CAUSAL O CASUAL? REPORTE DE UN CASO
}

\author{
Is aglossia adactylia syndrome and prenatal \\ exposure to misoprostol a causal or casual \\ relationship? A case report \\ Julián Ramírez-Cheyne*, Harry Pachajoa, M.D.**, Carolina Isaza, M.D., M.Sc.***, \\ Wilmar Saldarriaga, M.D., M.Sc. ****
}

Recibido: abril 8/08 - Aceptado: agosto 11/08

\section{RESUMEN}

Introducción y objetivo: el síndrome de aglosiaadactilia es un síndrome polimalformativo congénito de muy baja frecuencia, con una etiología y patrón de herencia que no son claros. Se hace una búsqueda bibliográfica orientada a la asociación entre la exposición prenatal a misoprostol y malformaciones congénitas.

Se presenta un caso de este síndrome asociado a exposición prenatal a misoprostol a las 10 semanas de gestación, asociación no establecida en la literatura revisada.

Presentación del caso: recién nacido con características fenotípicas de síndrome de aglosia-adactilia, hijo de madre de 17 años, con antecedente de haber utilizado misoprostol en dosis de 400 microgramos

* Médico interno, Escuela de Medicina, Universidad del Valle. Cali, Colombia.

** Médico, Aspirante a Doctor en Ciencias Biomédicas, Escuela de Ciencias Básicas Médicas, Universidad del Valle. Cali, Colombia. Correo electrónico: harrympl@yahoo.com

*** Profesora titular, Escuela de Ciencias Básicas Médicas, Universidad del Valle. Cali, Colombia.

**** Profesor asistente, Escuela de Ciencias Básicas Médicas, Escuela de Medicina, Universidad del Valle, Ginecólogo y obstetra, M.Sc. en Embriología y Genética. Fundación Valle del Lili. Hospital Universitario del Valle. Cali, Colombia. Grupo de Malformaciones Congénitas Perinatales y Dismorfología, Universidad del Valle. Cali, Colombia. vía oral y 200 microgramos vía vaginal a las 10 semanas de gestación, con el objetivo de interrumpir el embarazo.

Discusión: el misoprostol es un análogo sintético de la prostaglandina E1, se ha asociado a un aumento en el riesgo de la ocurrencia de secuencia de Moebius, artrogriposis, síndrome de aglosia-adactilia, defectos de las extremidades de tipo terminal y transversal en madres que utilizan este medicamento durante el primer trimestre de gestación.

Palabras clave: defectos congénitos, misoprostol, embarazo.

\section{SUMMARY}

Introduction and objective: the aglossia adactylia syndrome is an infrequently occurring congenital polymalformative syndrome, having unclear aetiology and inheritance pattern. A bibliographical search was carried out, orientated towards the association between the prenatal exposure to misoprostol and congenital malformations.

A case of this syndrome is presented which was associated with prenatal exposure to misoprostol during the 10th week of gestation; such associa- 
tion has not been established in the literature reviewed.

Presentation of the case: a newborn having phenotypical aglossia adactylia syndrome characteristics, the son of a 17-year-old mother having antecedents of having used a 400 microgram oral dose and a 200 microgram vaginal dose of misoprostol at 10 weeks' gestation with the aim of interrupting her pregnancy.

Discussion: misoprostol is a synthetic prostaglandin E1 analogue; it has been associated with increased risk of Moebius sequence occurring, arthrogryposis, aglossia adactylia syndrome and terminal and cross-sectional extremity defects in mothers who have used this drug during their first gestation trimester.

Key words: congenital defect, misoprostol, pregnancy.

\section{INTRODUCCIÓN}

El síndrome de aglosia-adactilia hace parte del espectro de la hipogénesis oromandibular y de las extremidades (MIM 103300), el cual se caracteriza por presentar malformaciones en los derivados del primer y segundo arco branquial y reducción de extremidades. Este síndrome es de baja prevalencia, con una etiología desconocida y probablemente heterogénea. ${ }^{1}$

La exposición prenatal a misoprostol se ha asociado a la ocurrencia de defectos congénitos, principalmente la secuencia de Moebius y defectos de las extremidades de tipo terminal y transversal., ${ }^{2,3}$ Sin embargo, la relación de causalidad entre misoprostol y malformaciones congénitas es discutida en la literatura. Por lo tanto, para el ginecobstetra es difícil establecer la probabilidad de aparición de MFC en un recién nacido $(\mathrm{RN})$ que fue expuesto a misoprostol en el primer trimestre del embarazo. ${ }^{2}$

Se reporta un caso con diagnóstico de síndrome de aglosia-adactilia asociado a exposición a misoprostol en la semana 10 de gestación, siendo éste el segundo reporte del mismo grupo de investigación. ${ }^{4}$

\section{REPORTE DE CASO}

Recién nacido de sexo masculino, parto atendido en el Hospital Universitario del Valle, hospital de nivel III de la ciudad de Cali en Colombia y el mayor centro de referencia de la red pública, que atiende principalmente a la población del régimen subsidiado y de bajos ingresos socioeconómicos. Apgar de 8 y 9 al primer y quinto minuto. Ballard para 37 semanas, peso: $2.600 \mathrm{~g}(\mathrm{p}=5-10)$, talla: $50 \mathrm{~cm}(\mathrm{p}=50)$, perímetro cefálico: $33 \mathrm{~cm}(\mathrm{p}=3-10)$, distancia entre los cantos internos: $22 \mathrm{~mm}$ (percentil $=50-75$ ), entre los cantos externos: $65 \mathrm{~mm}$ (percentil $=50-75$ ), filtrum: $8 \mathrm{~mm}$. En el examen físico se encontraron los siguientes hallazgos: pliegue epicántico bilateral, parálisis facial bilateral, paladar hendido, microstomía, frenillo lingual, hipoglosia (lengua hipoplásica), microstomía (figuras 1, 2); además presentaba sindactilia del tercer, cuarto y quinto dedo de la mano derecha, braquidactilia (dedos pequeños) y pie equinovaro derecho.

Figura 1. Paciente con espectro de la hipogénesis oromandibular y de las extremidades. Nótese la parálisis facial bilateral, la micrognatia y el pie equinovaro.

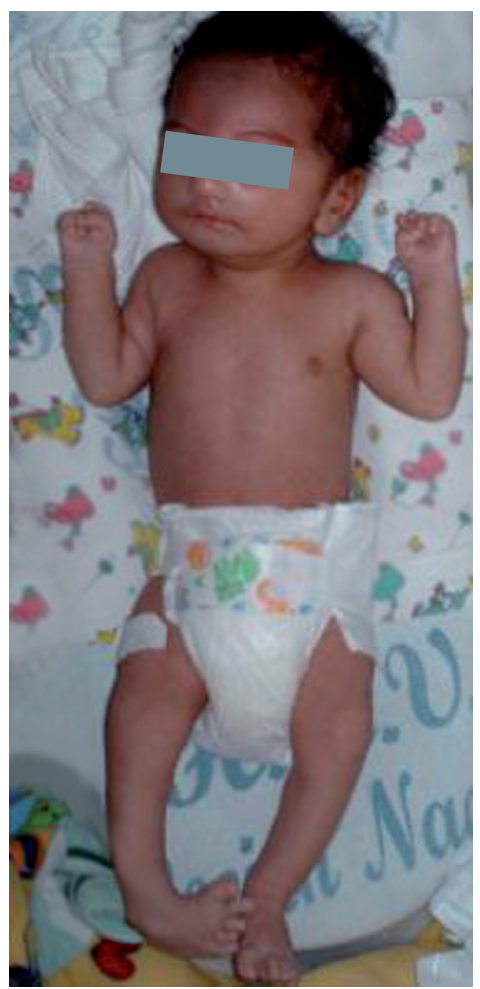


Figura 2. Nótese la micrognatia y la parálisis facial.

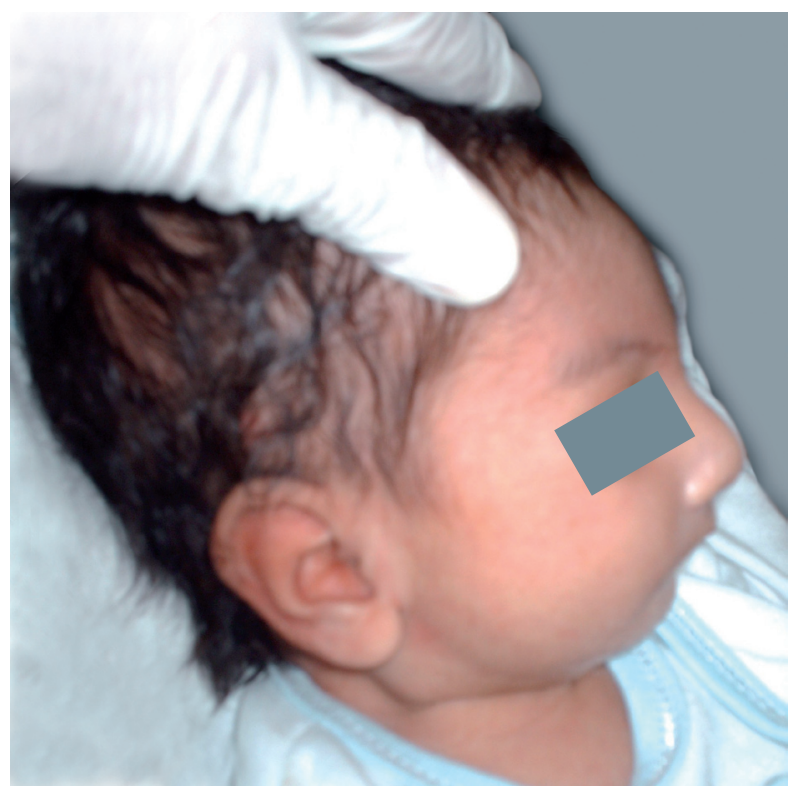

Hijo de padres no consanguíneos, sin antecedentes familiares sugestivos de enfermedad hereditaria, madre primigestante de 17 años, con antecedente de haber utilizado misoprostol en dosis de $400 \mathrm{mi}$ crogramos vía oral y 200 microgramos vía vaginal a las 10 semanas de gestación, con el objetivo de interrumpir el embarazo; presentó sangrado escaso, la gestación continuó sin otras complicaciones, se realizó ecografías en el segundo y tercer trimestre, las cuales no reportaron malformaciones.

Exámenes complementarios: ecografía transfontanelar normal.

\section{DISCUSIÓN}

El síndrome de aglosia-adactilia es un defecto congénito polimalformativo, con una prevalencia que se ha estimado en 1 de cada 175.000 nacimientos, afectando por igual a hombres y mujeres. ${ }^{5}$ La etiología es desconocida, probablemente heterogénea. Se han propuesto patrones de herencia autosómico dominante, ${ }^{1}$ autosómico recesivo, ${ }^{6}$ y se han reportado múltiples casos no relacionados. Otros sugieren su origen en una disrupción vascular. ${ }^{2}$

Las características fenotípicas del síndrome de aglosia-adactilia incluyen un espectro amplio, desde hipoglosia (lengua pequeña) y braquidactilia (dedos pequeños), hasta la ausencia completa de lengua (aglosia) y de dedos (adactlia), por lo cual se ha propuesto incluir este síndrome dentro del espectro de la hipogénesis oromandibular y de las extremidades. ${ }^{7}$

Además, se han encontrado en estos pacientes otras malformaciones como epicanto, telecanto, anomalías estructurales del oído medio y sordera conductiva, base nasal estrecha, microstomía, frenulum ventral anormal, paladar ojival, anodoncia, oligodoncia, retrognatia, micrognatia. Puede acompañarse también de retraso mental moderado a severo, asimetría facial, parálisis de pares craneales principalmente VI y VII y con menor frecuencia III, V y XII. ${ }^{1}$ El caso aquí reportado por nosotros presentó las características clásicas de este síndrome. Tabla 1.

El misoprostol (Cytotec ${ }^{\circledR}$ ) es un análogo sintético de la prostaglandina E1, aprobado por la FDA para la prevención y el tratamiento de úlceras gástricas asociadas al uso de antiinflamatorios no esteroideos por su efecto antisecretor de ácidos gástricos. Es clasificado como factor de riesgo categoría X en el embarazo por su efecto abortivo, ${ }^{8}$ por ser una prostaglandina, produce contracción del músculo liso uterino y separación de las fibras de colágeno existentes en el cuello uterino, y se usa para inducción del trabajo de parto en la práctica obstétrica. Debido a estos efectos, en países donde el aborto es ilegal, el misoprostol es utilizado como un abortivo, siendo menos costoso que otros métodos ilegales. ${ }^{9}$

En 1992, Schuler y colaboradores reportan 29 mujeres expuestas a misoprostol en el primer trimestre, encontrando 3 abortos, 3 fetos muertos y 17 recién nacidos $(\mathrm{RN})$ sin malformaciones. ${ }^{10}$ El mismo autor en 1999 reporta dos grupos de pacientes, de 67 mujeres expuestas a misoprostol, 2 tuvieron productos con malformaciones mayores, probablemente relacionadas a la exposición; y en un grupo de mujeres no expuestas, se encontraron 2 recién nacidos con malformaciones similares. ${ }^{11}$ 
Tabla 1. Características fenotípicas del espectro de la hipogénesis oromandibular y de las

$$
\text { extremidades. }
$$

\begin{tabular}{|c|c|c|}
\hline \multicolumn{2}{|c|}{$\begin{array}{l}\text { Malformaciones reportadas en } \\
\text { el espectro de la hipogénesis } \\
\text { oromandibular y de las extremidades }\end{array}$} & $\begin{array}{l}\text { Caracterís- } \\
\text { ticas encon- } \\
\text { tradas en } \\
\text { este } \\
\text { paciente }\end{array}$ \\
\hline \multirow{8}{*}{ Craneofaciales } & Hipoglosia & Sí \\
\hline & Microstomía & Sí \\
\hline & Micrognatia & Sí \\
\hline & $\begin{array}{c}\text { Parálisis } \\
\text { de nervios } \\
\text { craneales } \\
\text { (secuencia de } \\
\text { Moebius) }\end{array}$ & Sí \\
\hline & $\begin{array}{l}\text { Paladar } \\
\text { hendido }\end{array}$ & Sí \\
\hline & $\begin{array}{l}\text { Hipodoncia } \\
\text { mandibular }\end{array}$ & $\begin{array}{c}\text { No evaluada } \\
\text { por la edad }\end{array}$ \\
\hline & Telecanto & No \\
\hline & Epicanto & Sí \\
\hline \multirow{4}{*}{ Extremidades } & Adactilia & No \\
\hline & $\begin{array}{l}\text { Hipodactilia - } \\
\text { Braquidactilia }\end{array}$ & Sí \\
\hline & Sindactilia & Sí \\
\hline & Ectrodactilia & No \\
\hline Otras & $\begin{array}{l}\text { Malformacio- } \\
\text { nes cerebrales }\end{array}$ & No \\
\hline
\end{tabular}

Sin embargo, se han hecho múltiples reportes de exposición e este fármaco en el primer trimestre del embarazo y la ocurrencia de defectos congénitos, como la secuencia de Moebius, artrogriposis, defectos de las extremidades de tipo terminal y transversal. ${ }^{12,13}$ Estos defectos serían el producto de la rotura de las membranas amnióticas o una disrupción vascular. ${ }^{3,14-17}$

Si bien los RN expuestos a misoprostol en el primer trimestre del embarazo tienen mayor probabilidad de presentar las malformaciones descritas, no es claro qué porcentaje del total de embriones o fetos expuestos tendrán malformaciones, puesto que la mayoría de los casos descritos son reportes únicos o series de casos.
Basados en el hallazgo del caso aquí descrito, la explicación fisiopatogénica del mecanismo de acción teratogénica del misoprostol y el probable origen vascular del síndrome de aglosia-adactilia, proponemos que existe una probable relación causal entre el consumo de misoprostol en el primer trimestre del embarazo y el síndrome de aglosia-adactilia, ${ }^{18,19}$ hallazgo que deberá ser confirmado mediante estudios epidemiológicos con un diseño adecuado para evaluar la causalidad.

\section{Consideraciones éticas}

Las fotos aquí expuestas son publicadas con autorización de los familiares.

\section{REFERENCIAS}

1. Online Mendelian Inheritance in Man, OMIM (TM). Johns Hopkins University, Baltimore, MD. MIM Number: 103300: Date last edited: 07/07/2003. Disponible en:http://www.ncbi.nlm.nih.gov/omim/

2. Vargas FR, Schuler-Faccini L, Brunoni D, Kim C, Meloni VF, Sugayama SM, et al. Prenatal exposure to misoprostol and vascular disruption defects: a casecontrol study. Am J Med Genet 2000;95:302-6.

3. da Silva Dal Pizzol T, Knop FP, Mengue SS. Prenatal exposure to misoprostol and congenital anomalies: systematic review and meta-analysis. Reproductive Toxicology 2006;22:666-71.

4. Isaza C, Saldarriaga W, García, R, López MC, Suárez F, Zarante I. Riesgos del misoprostol cuando falla como abortivo en el primer trimestre. Salud UIS 2006;38:66-70.

5. Firth HV, Boyd PA, Chamberlain PF, MacKenzie IZ, Morriss-Kay GM, Huson SM. Analysis of limb reduction defects in babies exposed to chorionic villus sampling. Lancet 1994;343:1069-71.

6. Tuncbilek E, Yalcin C, Atasu M. Aglossia-adactylia syndrome (special emphasis on the inheritance pattern). Clin Genet 1977;11:421-3.

7. Jones K. Smith's recognizable patterns of human malformations. 6th ed. Philadelphia: W.B. Elselvier Saunders Co; 2007. p. 724-43

8. Norman JE, Thong KG, Baird DT. Uterine contractility and induction of abortion in early pregnancy by misoprostol and mifepristone. Lancet 1991;338:1233-6. 
9. Costa SH. Commercial availability of misoprostol and induced abortion in Brazil. Int J Gynaecol Obstet 1998;63:S131-9.

10. Schüler L, Ashton PW, Sanseverino MT. Teratogenicity of misoprostol. Lancet 1992;339:437-8.

11. Schüler L, Pastuszak A, Sanseverino TV, Orioli IM, Brunoni D, Ashton-Prolla P, et al. Pregnancy outcome after exposure to misoprostol in Brazil: a prospective, controlled study. Reprod Toxicol 1999; 13:147-51

12. Vargas FR, Schuler-Faccini L, Brunoni D, Kim C, Meloni VF, Sugayama SM, et al. Prenatal exposure to misoprostol and vascular disruption defects: a casecontrol study. Am J Med Genet 2000;95:302-6.

13. da Silva Dal Pizzol T, Knop FP, Mengue SS. Prenatal exposure to misoprostol and congenital anomalies: systematic review and meta-analysis. Reprod Toxicol 2006;22:666-71.

14. Fonseca W, Alencar AJ, Mota FS, Coelho HL. Misoprostol and congenital malformations. Lancet 1991;338:56.
15. González CH, Vargas FR, Pérez AB, Kim CA, Brunoni D, Marques-Dias MJ, et al. Limb deficiency with or without Möbius sequence in seven Brazilian children associated with misoprostol use in first trimester of pregnancy. Am J Med Genet 1993;47:59-64.

16. González CH, Marques-Dias MJ, Kim CA, Sugayama SM, Da Paz JA, Huson SM, et al. Congenital abnormalities in Brazilian children associated with misoprostol misuse in first trimestre of pregnancy. Lancet 1998;351:1624-7.

17. Robinow M, Marsh JL, Edgerton MT, Sabio H, Johnson GF. Discordance in monozygotic twins for aglossia-adactylia, and possible clues to the pathogenesis of the syndrome. Birth Defects Orig Art Ser 1978;14:223-30.

18. Shepard TH. Möbius syndrome after misoprostol: a possible teratogenic mechanism. Lancet 1995;346:780.

19. Fawcett LB, Buck SJ, Brent RL. Limb reduction defects in the $\mathrm{A} / \mathrm{J}$ mouse strain associated with maternal blood loss. Teratology 1998;58:183-9. 